Review Article

\title{
Importance of Diversity in the Oral Microbiota including Candida Species Revealed by High-Throughput Technologies
}

\author{
Tamaki Cho, Jun-ichi Nagao, Rieko Imayoshi, and Yoshihiko Tanaka \\ Section of Infection Biology, Department of Functional Bioscience, Fukuoka Dental College, 2-15-1 Tamura, Sawara-ku, \\ Fukuoka 814-0193, Japan \\ Correspondence should be addressed to Tamaki Cho; chot1@college.fdcnet.ac.jp
}

Received 31 January 2014; Accepted 1 April 2014; Published 22 April 2014

Academic Editor: Masahiro Yoneda

Copyright (c) 2014 Tamaki Cho et al. This is an open access article distributed under the Creative Commons Attribution License, which permits unrestricted use, distribution, and reproduction in any medium, provided the original work is properly cited.

Taking advantage of high-throughput technologies, deep sequencing of the human microbiome has revealed commensal bacteria independent of the ability to culture them. The composition of the commensal microbiome is dependent on bacterial diversity and the state of the host regulated by the immune system. Candida species are well known as components of the commensal oral microbiota. Candida species frequently colonize and develop biofilms on medical devices like dentures and catheters. Therefore, Candida biofilm on dentures leads to a decrease in the bacterial diversity and then to a change in the composition of the oral microbiota. A disturbance in the balance between commensal bacteria and the host immune system results in a switch from a healthy state to a diseased state even in the limited oral niche.

\section{Introduction}

The progression of a global aging population has been accompanied by severe problems in oral health because aging induces risk factors like tooth loss, wearing dentures, senescence of tissues, and systemic diseases (diabetes, malignancies, implantations of tissues, etc.), which may disturb the oral homeostasis [1-10]. Candida species, especially Candida albicans, is a normal component of the human flora. However, C. albicans causes oral candidiasis in immunocompromised hosts [11-13]. On the other hand, periodontitis is also induced by a change in the balance between oral bacteria and the host defense mechanisms [14-16]. The role of Candida species in the oral microbiota has not been well studied. Here, as shown in Figure 1, the presence of Candida species and the pathogens of periodontitis in generally healthy subjects is described. Moreover, the microbiology of periodontitis including Candida species in HIV infected patients is described.

\section{Microbiome and Mycobiome in the Oral Cavity}

Normal control samples are necessary to determine whether there are associations between changes in the microbiome and health/disease and also to yield insights into the role of the endogenous flora in health and disease [17-19]. The NIH Human Microbiome project was funded to take advantage of high-throughput technologies using metagenomic sequencing approaches to characterize the human microbiome on multiple body sites (the gastrointestinal (GI) tract, the mouth, the vagina, the skin, and the nasal cavity) from healthy volunteers [20-22]. The distribution by body site of bacteria in the NIH project [20] showed that the distribution on the oral sites (buccal mucosa, hard palate, keratinized gingiva, palatine tonsils, saliva, sub- and supragingival plaques, throat, and tongue dorsum) was $26 \%$ of the total $(n=250)$.

The oral cavity is comprised of many microbial habitats, such as teeth, gingival sulcus, attached gingiva, tongue, cheek, lip, and hard and soft palates. Mager et al. [23] analyzed the proportion of 40 bacterial species on different intraoral surfaces of 225 healthy subjects using checkerboard DNADNA hybridization. The proportions of bacterial species differed markedly on different intraoral surfaces. For example, Actinomyces spp. colonized teeth at higher proportions than soft tissues. The microbiota of saliva was most similar to that of the dorsal and lateral surfaces of the tongue. Prevotella melaninogenica, Veillonella parvula, and Streptococcus mitis 


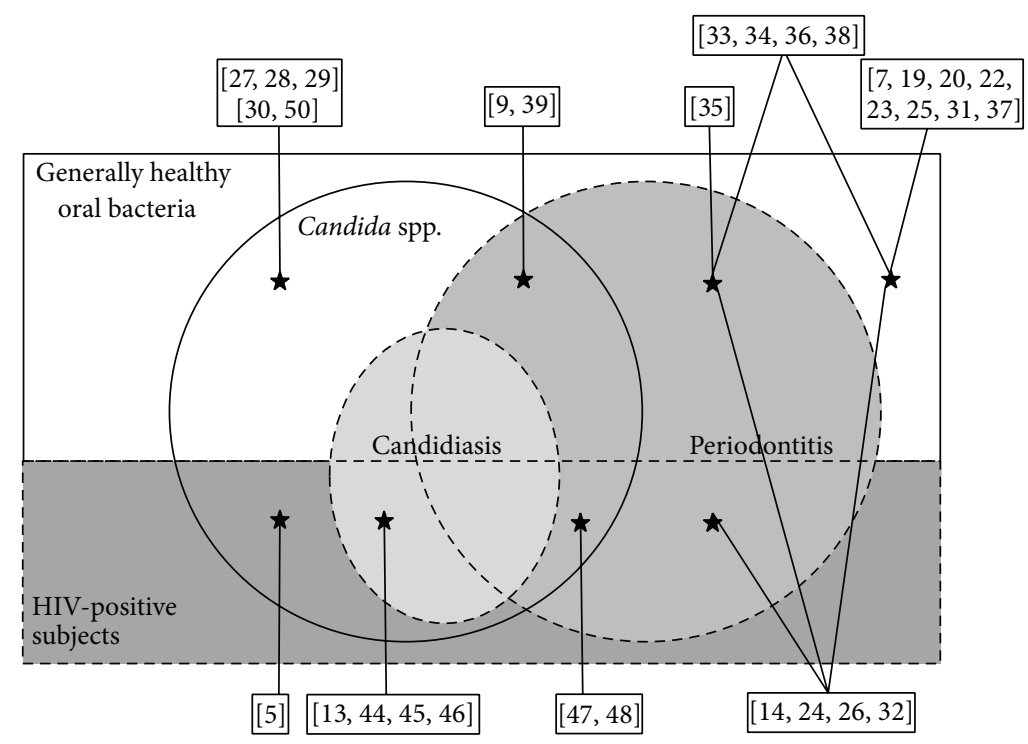

FIgURE 1: The microbiota and diseases described in the literature are cited in this review. The white background represents oral bacteria from generally healthy subjects. The dark grey background represents oral bacteria from HIV-positive subjects. The circle of Candida spp. represents detection of Candida spp. from oral bacteria in subjects. The light gray circle of candidiasis represents subjects with candidiasis. The gray circle of periodontitis represents subjects with periodontitis. Numbers in squares represent number of references cited in the text.

were found in higher proportions on soft tissue surfaces. Paster et al. [24] estimated 500 species in subgingival plaque of periodontally healthy subjects $(n=2)$, periodontitis subjects $(n=9)$, HIV periodontitis patients $(n=2)$, and acute necrotizing ulcerative gingivitis subjects $(n=4)$ using culture-independent molecular techniques (16S rRNA cloning). Also they reported that known putative periodontal pathogens such as Porphyromonas gingivalis and Bacteroides forsythus (Tannerella forsythia) were identified from multiple subjects, but as minor components of the plaque as seen in cultivable studies. Aas et al. [25] defined the human microflora of the healthy oral cavity. The breadth of bacterial diversity in the oral cavity (dorsum of the tongue, lateral sides of the tongue, buccal fold, hard and soft palates, labial gingiva, tonsils, and supra- and subgingival plaques from tooth surfaces) in 5 healthy subjects was analyzed using 16S rRNA identification. It was reported that species common to all oral sites belonged to the genera Gemella, Granulicatella, Streptococcus, and Veillonella. Moreover, they identified 13 new phylotypes. A distinctive predominant bacterial flora of the healthy oral cavity was highly diverse and site and subject specific. As much as $60 \%$ of the species detected have not been cultivated.

Dewhirst et al. [26] established the Human Oral Microbiome Database (HOMD) based on analysis of 16S rRNA gene clone libraries created in published and unpublished studies from oral health and disease statuses including periodontitis, HIV periodontitis, acute necrotizing ulcerative gingivitis, caries, endodontic infections, and NOMA. The analysis identified 1,179 taxa, of which $24 \%$ were named, $8 \%$ were cultivated but unnamed, and $68 \%$ were uncultivated phylotypes.
Although the oral microbiome has been analyzed in detail, Ghannoum et al. [27] first characterized the fungi present in the oral cavity of 20 healthy subjects using a novel multitag pyrosequencing approach with the pan-fungal internal transcribed spacer (ITS) primers. Sampling was obtained by oral rinses with phosphate buffered saline. The collection of organisms from the dorsum of the tongue and the oral mucosal environment was possible by rinsing. Candida species were isolated from $75 \%$ of all study participants (21-60 years of age). Although environmental fungi such as Cladosporium, Aureobasidium, Saccharomycetales, Aspergillus, Fusarium, and Cryptococcus were unexpected in the oral cavity of healthy individuals, they were detected in the oral wash samples. These could simply be spores inhaled from the air or material ingested with food.

Denture wearers can present an increase in the number of Candida species cells [28, 29]. Kraneveld et al. [30] studied the relationship between the Candida load and the bacterial microbiome profiles of saliva of elderly Dutch adults. Unstimulated saliva was collected for 5 minutes from 82 adults (5880 years of age). The subjects included a dentate with or without partial prosthesis and an edentate with full upper and lower dentures. Ninety-seven \% of the subjects were positive for the Candida-specific internal transcribed spacer (ITS) gene. There was a negative correlation between the Candida load and the bacterial profiles of saliva. With increased Candida load the diversity of the salivary microbiome decreased and the composition changed towards dominance by streptococci and lactobacilli and the disappearance of genera within Fusobacteria and Bacteroidia classes. Therefore, decreased bacterial diversity could be associated with a disbalanced community. 


\section{Oral Microbiota including Candida Species in Periodontitis Patients and HIV Infected Patients}

Simon-Soro et al. [31] reported an interesting study regarding sampling sites from oral cavity. They determined the bacterial diversity of different oral microniches of 2 healthy volunteers. Samples were taken from each vestibular and lingual surface of the individual's teeth and gingival sulcus, as well as samples from the tongue dorsum and nonstimulated and stimulated saliva. Interestingly, streptococci are found at higher proportions on the vestibular sulcus compared with lingual sulcus, whereas the highest proportions of obligate anaerobes like Fusobacterium were at lingual sites. Nonstimulated saliva samples were not representative of supra- and subgingival plaque. The results from saliva sample accord with the results of Mager et al. [23].

Liu et al. [32] analyzed the oral microbiome in supraand subgingival plaques of 3 healthy individuals and 2 periodontitis patients who were in good general health. In healthy samples, Gram-positive genera Streptococcus, Actinomyces, and Granulicatella were significantly enriched; however, Fusobacterium and Porphyromonas were not predominantly in the periodontal disease samples. A microbiota of one of 3 healthy subjects resembled the diseased samples. The control subjects exhibited mild bleeding at probing time, but no attachment loss. Therefore, the control subject could be in initial periodontal disease. The authors suggested that it is necessary to analyze carefully the clinical data collected during sampling. Also the observation may indicate that the microbiota shifts into a disease state before the full clinical symptoms of the disease are apparent. Moreover, Kumar et al. [33] assessed subgingival plaque samples by cloning and sequencing 16S rRNA genes and proved that changes in periodontal health status were associated with bacterial community shifts. Therefore, we understand that the bacterial community is changed from periodontal health to periodontal disease. However, the relative contributions of the host immune system and the shift of the bacterial community in clinically healthy states as suggested by Liu et al. [32] are not well known. Darveau [34] also pointed out the importance of investigating the mechanisms that maintain the stability of, or induce changes in, the microbial composition. Faveri et al. [35] researched the microbiological diversity in subgingival plaque samples from subjects with early onset periodontitis using $16 \mathrm{~S}$ rRNA clonal analysis. Selenomonas and Streptococcus accounted for 50\% of the clone libraries from the plaque. Therefore, the results showed microbiological diversity in the plaque was altered and decreased. Divaris et al. [36] and Wade [37] insisted that an understanding of host-microbiome interactions is highly important because periodontitis is not an infectious disease in the classical sense, but results from a complex interaction between the commensal microbiota, host susceptibility, and environmental factors. For example, Ge et al. [38] studied factors including smoking, race, and dental caries that may influence subgingival bacterial diversity. Periodontal disease and these influences were found to be independent.
McManus et al. [39] investigated whether clades (a group composed of one ancestor and its descendants) of C. albicans isolated from periodontal pockets and periodontal healthy subgingival sites in patients with untreated periodontitis were associated with periodontitis. Specific clonal groups were analyzed by the multilocus sequence typing (MLST) method [40-42]. C. albicans was isolated from samples of periodontal pockets, healthy subgingival sites, and oral rinses from untreated periodontal subjects. Thirty-one isolates from periodontal pockets belonged to 19 sequence typings, with 11 isolates belonging to MLST clade 1. Sixteen C. albicans isolates from oral rinses of healthy subjects belonged to 16 sequence types, with 4 isolates belonging to clade 1 . The distributions of sequence types between healthy subjects and periodontitis patients were significantly different. According to other reports, virulence factors and sites of isolation are associated with clade specificity $[42,43]$. However, it is not clear if the presence of $C$. albicans in the periodontal pockets contributes to the progression of periodontitis.

The rate of oral C. albicans carriage in HIV-positive subjects is higher than in control subjects, and patients with CD4+-cell counts of 200-400/microliter had a significantly higher level of yeast carriage [44]. The samples were collected from the buccal mucosa, the floor of the mouth, and the dorsal surface using a sterile cotton swab. C. albicans was the most prevalent agent of oral candidiasis in HIV-positive subjects from resource-rich countries [45]. Oral Candida colonization was not affected with antiretroviral therapy in HIV-positive patients [46]. Moreover, C. albicans was recovered most often from oral and subgingival samples in HIV-positive subjects with advanced periodontal disease [47]. There is an increase in the prevalence of Candida species in the subgingival plaque of HIV-seropositive patients compared to immunocompetent subjects [48]. Aas et al. [49] compared the predominant bacterial and fungal species associated with gingivitis $(n=5)$, periodontitis $(n=8)$, and linear gingival erythema (LEG [50], $n=1$ ) in 14 HIV-positive subjects. Samples were isolated with cotton rolls from supraand subgingival plaque. The classical periodontal pathogens, Treponema denticola, P. gingivalis, and T. forsythia, were not detected. The predominant bacterial species were potential opportunistic pathogens, Gemella, Dialister, Streptococcus, and Veillonella. In 2 of 4 subjects with low viral loads, high CD4 levels, and periodontitis, C. albicans was predominant and $S$. cerevisiae was only a minor component. However, $S$. cerevisiae (not $C$. albicans) was the only fungal species detected from the LGE subject and 2 of 4 severe subjects with high viral loads, low CD4 levels, and periodontitis. These data were indicative of opportunistic infection in a highly susceptible immunocompromised host.

\section{Conclusion}

The oral healthy state is in optimum balance between the oral microbiota and the host immune system (Figure 2). The healthy state maintains the diversity of the oral microbiota. A reduction in diversity such as Candida biofilm formation on dentures may respond to the host immune system, resulting 


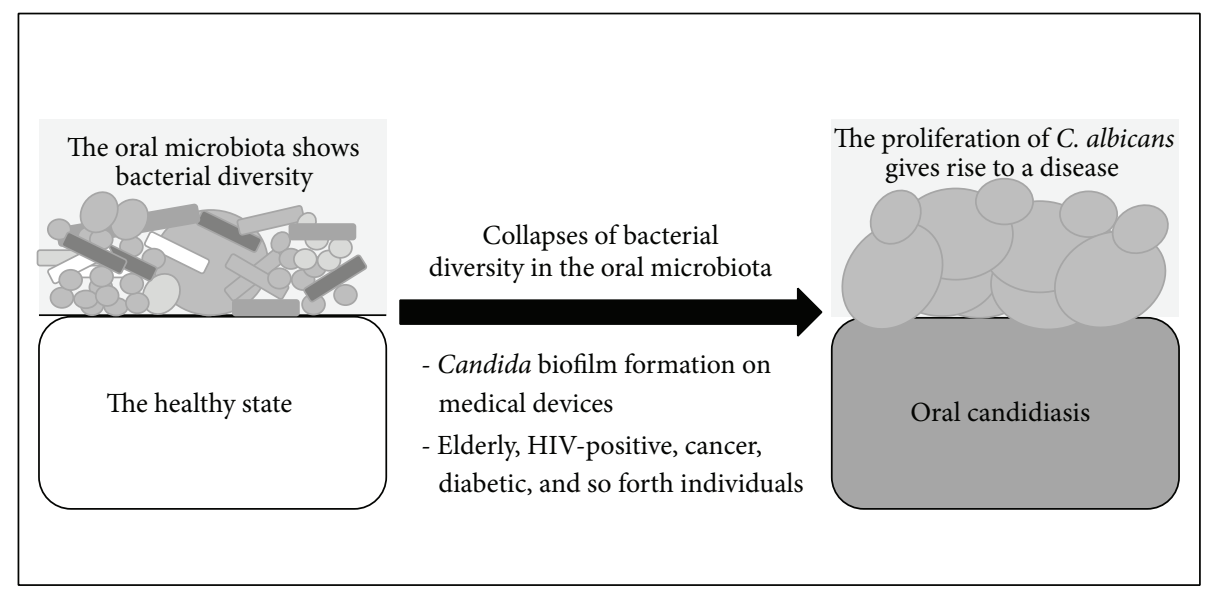

FIGURE 2: Illustration of the shift in the commensal microbiota in the healthy state relative to the disease state (see Section 4).

in the shift into a disease state though the symptoms of the disease are not always apparent.

\section{Conflict of Interests}

The authors declare that there is no conflict of interests regarding the publication of this paper.

\section{References}

[1] A. Gupta, J. B. Epstein, and H. Sroussi, "Hyposalivation in elderly patients," Journal of the Canadian Dental Association, vol. 72, no. 9, pp. 841-846, 2006.

[2] K. Fujihashi and J. R. McGhee, "Mucosal immunity and tolerance in the elderly," Mechanisms of Ageing and Development, vol. 125, no. 12, pp. 889-898, 2004.

[3] K. Kampoo, R. Teanpaisan, R. G. Ledder, and A. J. McBain, "Oral bacterial communities in individuals with type 2 diabetes who live in southern Thailand," Applied and Environmental Microbiology, vol. 80, no. 2, pp. 662-671, 2014.

[4] A. Surdacka, E. Cięzka, M. Pioruńska-Stolzmann et al., "Relation of salivary antioxidant status and cytokine levels to clinical parameters of oral health in pregnant women with diabetes," Archives of Oral Biology, vol. 56, no. 5, pp. 428-436, 2011.

[5] D. Merenstein, H. Hu, C. Wang et al., "Colonization by Candida species of the oral and vaginal mucosa in HIV-infected and noninfected women," AIDS Research and Human Retroviruses, vol. 29, no. 1, pp. 30-34, 2013.

[6] W. C. Gonsalves, A. S. Wrightson, and R. G. Henry, "Common oral conditions in older persons," American Family Physician, vol. 78, no. 7, pp. 845-852, 2008.

[7] E. Brasili, E. Mengheri, A. Tomassini et al., "Lactobacillus acidophilus $\mathrm{La} 5$ and Bifidobacterium lactis Bb12 induce different age-related metabolic profiles revealed by $1 \mathrm{H}-\mathrm{NMR}$ spectroscopy in urine and feces of mice," The Journal of Nutrition, vol. 143, no. 10, pp. 1549-1557, 2013.

[8] M. Soell, M. Hassan, A. Miliauskaite, Y. Haïkel, and D. Selimovic, "The oral cavity of elderly patients in diabetes," Diabetes \& Metabolism, vol. 33, supplement 1, pp. S10-S18, 2007.

[9] J. C. O. Sardi, C. Duque, J. F. Hofling, and R. B. Goncalves, "Genetic and phenotypic evaluation of Candida albicans strains isolated from subgingival biofilm of diabetic patients with chronic periodontitis," Medical Mycology, vol. 50, no. 5, pp. 467475, 2012.

[10] M. M. Hammad, A. M. Darwazeh, and M. M. Idrees, "The effect of glycemic control on Candida colonization of the tongue and the subgingival plaque in patients with type II diabetes and periodontitis," Oral Surgery, Oral Medicine, Oral Pathology, Oral Radiology, vol. 116, no. 3, pp. 321-326, 2013.

[11] D. L. Moyes and J. R. Naglik, "Mucosal immunity and Candida albicans infection," Clinical and Developmental Immunology, vol. 2011, Article ID 346307, 9 pages, 2011.

[12] D. W. Williams, R. P. C. Jordan, X. Wei et al., "Interactions of Candida albicans with host epithelial surfaces," Journal of Oral Microbiology, vol. 5, Article ID 22434, 2013.

[13] S. A. Khan, P. L. Fidel Jr., A. A. Thunayyan, S. Varlotta, T. F. Meiller, and M. A. Jabra-Rizk, "Impaired histatin-5 levels and salivary antimicrobial activity against C. albicans in HIV infected individuals," Journal of AIDS \& Clinical Research, vol. 4, no. 193, Article ID 1000193, 2013.

[14] M. T. Pollanen, M. A. Laine, R. Ihalin, and V.-J. Uitto, "Hostbacteria crosstalk at the dentogingival junction," International Journal of Dentistry, vol. 2012, Article ID 821383, 14 pages, 2012.

[15] G. Hajishengallis, "Complement and periodontitis," Biochemical Pharmacology, vol. 80, no. 12, pp. 1992-2001, 2010.

[16] K. Asif and S. V. Kothiwale, "Phagocytic activity of peripheral blood and crevicular phagocytes in health and periodontal disease," Journal of the Indian Society of Periodontology, vol. 14, no. 1, pp. 8-11, 2010.

[17] D. A. Relman and S. Falkow, "The meaning and impact of the human genome sequence for microbiology," Trends in Microbiology, vol. 9, no. 5, pp. 206-208, 2001.

[18] D. A. Relman, "New technologies, human-microbe interactions, and the search for previously unrecognized pathogens," The Journal of Infectious Diseases, vol. 186, supplement 2, pp. S254-S258, 2002.

[19] I. Nasidze, J. Li, D. Quinque, K. Tang, and M. Stoneking, "Global diversity in the human salivary microbiome," Genome Research, vol. 19, no. 4, pp. 636-643, 2009.

[20] The NIH HMP Working Group, "The NIH human microbiome project," Genome Research, vol. 19, no. 12, pp. 2317-2323, 2009. 
[21] The Human Microbiome Project Consortium, "A framework for human microbiome research," Nature, vol. 486, pp. 215-221, 2012.

[22] N. Segata, S. K. Haake, P. Mannon et al., "Composition of the adult digestive tract bacterial microbiome based on seven mouth surfaces, tonsils, throat and stool samples," Genome Biology, vol. 13, no. 6, article R42, 2012.

[23] D. L. Mager, L. A. Ximenez-Fyvie, A. D. Haffajee, and S. S. Socransky, "Distribution of selected bacterial species on intraoral surfaces," Journal of Clinical Periodontology, vol. 30, no. 7, pp. 644-654, 2003.

[24] B. J. Paster, S. K. Boches, J. L. Galvin et al., "Bacterial diversity in human subgingival plaque," Journal of Bacteriology, vol. 183, no. 12, pp. 3770-3783, 2001.

[25] J. A. Aas, B. J. Paster, L. N. Stokes, I. Olsen, and F. E. Dewhirst, "Defining the normal bacterial flora of the oral cavity," Journal of Clinical Microbiology, vol. 43, no. 11, pp. 5721-5732, 2005.

[26] F. E. Dewhirst, T. Chen, J. Izard et al., "The human oral microbiome," Journal of Bacteriology, vol. 192, no. 19, pp. 50025017, 2010.

[27] M. A. Ghannoum, R. J. Jurevic, P. K. Mukherjee et al., "Characterization of the oral fungal microbiome (mycobiome) in healthy individuals," PLoS Pathogens, vol. 6, no. 1, Article ID e1000713, 2010.

[28] J. M. R. Gusmão, S. S. F. dos Santos, M. P. Neisser, A. O. C. Jorge, and M. I. Faria, "Correlation between factors associated with the removable partial dentures use and Candida spp. in saliva," Gerodontology, vol. 28, no. 4, pp. 283-288, 2011.

[29] A. Vanden Abbeele, H. de Meel, M. Ahariz, J.-P. Perraudin, I. Beyer, and P. Courtois, "Denture contamination by yeasts in the elderly, Gerodontology, vol. 25, no. 4, pp. 222-228, 2008.

[30] E. A. Kraneveld, M. J. Buijs, M. J. Bonder et al., "The relation between oral Candida load and bacterial microbiome profiles in Dutch older adults," PLoS ONE, vol. 7, no. 8, Article ID e42770, 2012.

[31] A. Simon-Soro, I. Tomas, R. Cabrera-Rubio, M. D. Catalan, B. Nyvad, and A. Mira, "Microbial geography of the oral cavity," Journal of Dental Research, vol. 92, no. 7, pp. 616-621, 2013.

[32] B. Liu, L. L. Faller, N. Klitgord et al., "Deep sequencing of the oral microbiome reveals signatures of periodontal disease," PLoS ONE, vol. 7, no. 6, Article ID e37919, 2012.

[33] P. S. Kumar, E. J. Leys, J. M. Bryk, F. J. Martinez, M. L. Moeschberger, and A. L. Griffen, "Changes in periodontal health status are associated with bacterial community shifts as assessed by quantitative $16 \mathrm{~S}$ cloning and sequencing," Journal of Clinical Microbiology, vol. 44, no. 10, pp. 3665-3673, 2006.

[34] R. P. Darveau, "Periodontitis: a polymicrobial disruption of host homeostasis," Nature Reviews Microbiology, vol. 8, no. 7, pp. 481490, 2010.

[35] M. Faveri, M. P. A. Mayer, M. Feres, L. C. de Figueiredo, F. E. Dewhirst, and B. J. Paster, "Microbiological diversity of generalized aggressive periodontitis by $16 \mathrm{~S}$ rRNA clonal analysis," Oral Microbiology and Immunology, vol. 23, no. 2, pp. 112-118, 2008.

[36] K. Divaris, K. L. Monda, K. E. North et al., "Genome-wide association study of periodontal pathogen colonization," Journal of Dental Research, vol. 91, supplement 7, pp. 21S-28S, 2012.

[37] W. G. Wade, "The oral microbiome in health and disease," Pharmacological Research, vol. 69, no. 1, pp. 137-143, 2013.

[38] X. Ge, R. Rodriguez, M. Trinh, J. Gunsolley, and P. Xu, "Oral microbiome of deep and shallow dental pockets in chronic periodontitis," PLoS ONE, vol. 8, no. 6, Article ID e65520, 2013.
[39] B. A. McManus, R. Maguire, P. J. Cashin et al., "Enrichment of multilocus sequence typing clade 1 with oral Candida albicans isolates in patients with untreated periodontitis," Journal of Clinical Microbiology, vol. 50, no. 10, pp. 3335-3344, 2012.

[40] A. Tavanti, A. D. Davidson, M. J. Fordyce, N. A. R. Gow, M. C. J. Maiden, and F. C. Odds, "Population structure and properties of Candida albicans, as determined by multilocus sequence typing," Journal of Clinical Microbiology, vol. 43, no. 11, pp. 56015613, 2005.

[41] F. C. Odds, M.-E. Bougnoux, D. J. Shaw et al., "Molecular phylogenetics of Candida albicans," Eukaryotic Cell, vol. 6, no. 6, pp. 1041-1052, 2007.

[42] F. C. Odds, "In Candida albicans, resistance to flucytosine and terbinafine is linked to MAT locus homozygosity and multilocus sequence typing clade 1," FEMS Yeast Research, vol. 9, no. 7, pp. 1091-1101, 2009.

[43] J. P. Lyon, K. C. M. Moraes, L. M. Moreira, F. Aimbire, and M. A. de Resende, "Candida albicans: genotyping methods and clade related phenotypic characteristics," Brazilian Journal of Microbiology, vol. 41, no. 4, pp. 841-849, 2010.

[44] K. G. Vargas and S. Joly, "Carriage frequency, intensity of carriage, and strains of oral yeast species vary in the progression to oral candidiasis in human immunodeficiency virus-positive individuals," Journal of Clinical Microbiology, vol. 40, no. 2, pp. 341-350, 2002.

[45] F. Kwamin, N. O. Nartey, F. S. Codjoe, and M. J. Newman, "Distribution of Candida species among HIV-positive patients with oropharyngeal candidiasis in Accra, Ghana," The Journal of Infection in Developing Countries, vol. 7, no. 1, pp. 41-45, 2013.

[46] V. Maurya, A. Srivastava, J. Mishra et al., "Oropharyngeal candidiasis and Candida colonization in HIV positive patients in northern India," The Journal of Infection in Developing Countries, vol. 7, no. 8, pp. 608-613, 2013.

[47] M. A. Jabra-Rizk, S. M. S. Ferreira, M. Sabet, W. A. Falkler, W. G. Merz, and T. F. Meiller, "Recovery of Candida dubliniensis and other yeasts from human immunodeficiency virus-associated periodontal lesions," Journal of Clinical Microbiology, vol. 39, no. 12, pp. 4520-4522, 2001.

[48] L. Feller and J. Lemmer, "Necrotizing periodontal diseases in HIV-seropositive subjects: pathogenic mechanisms," Journal of the International Academy of Periodontology, vol. 10, no. 1, pp. 10-15, 2008.

[49] J. A. Aas, S. M. Barbuto, T. Alpagot, I. Olsen, F. E. Dewhirst, and B. J. Paster, "Subgingival plaque microbiota in HIV positive patients," Journal of Clinical Periodontology, vol. 34, no. 3, pp. 189-195, 2007.

[50] L. P. Samaranayake, W. K. Leung, and L. Jin, "Oral mucosal fungal infections," Periodontology 2000, vol. 49, no. 1, pp. 39-59, 2009. 


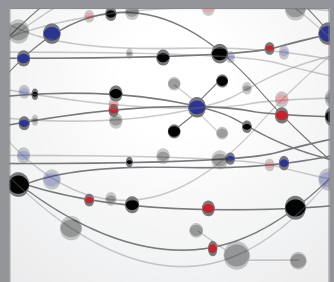

The Scientific World Journal
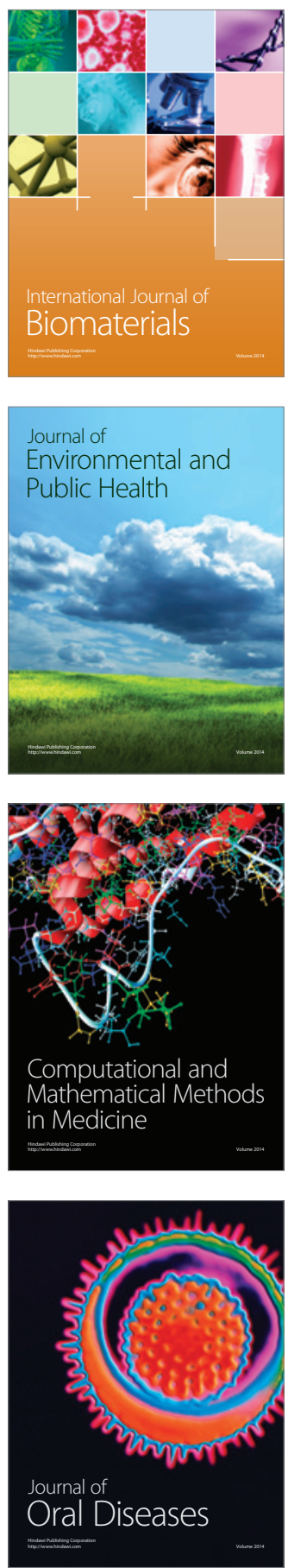
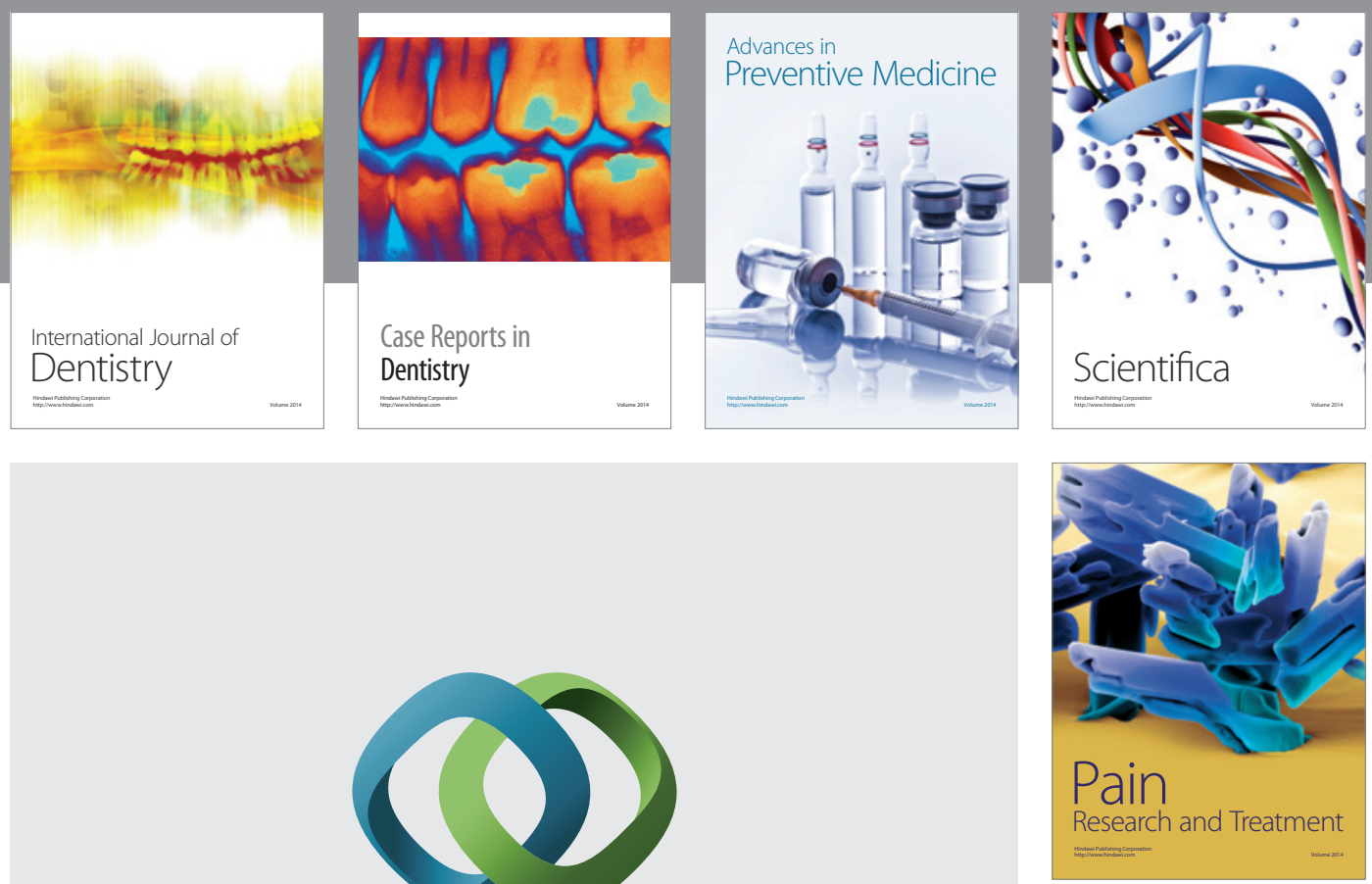

\section{Hindawi}

Submit your manuscripts at

http://www.hindawi.com
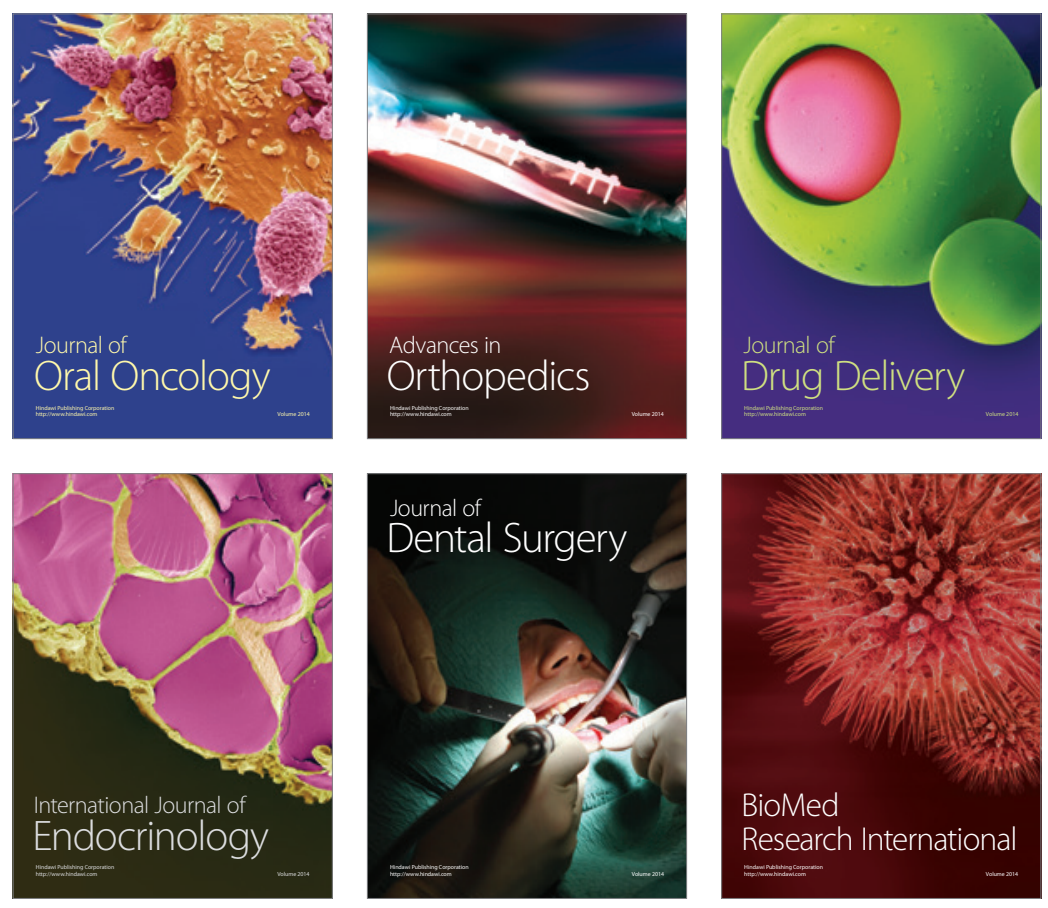

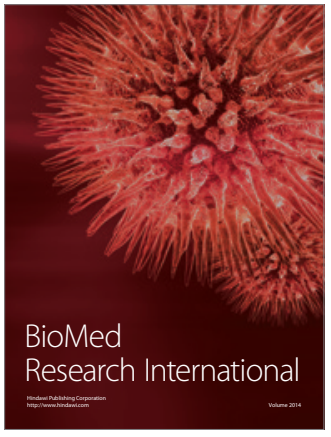

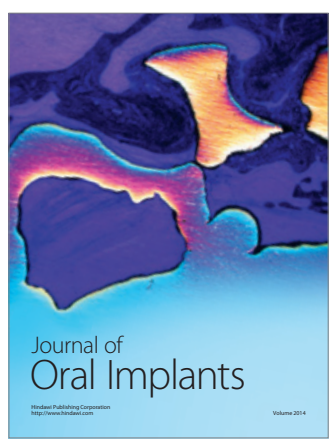
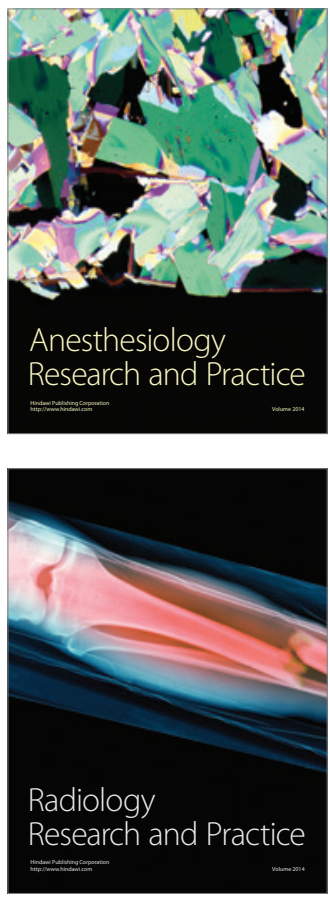